\title{
Renormalization group flow and parallel transport with non-metric compatible connections
}

\author{
Brian P. Dolan ${ }^{\mathrm{a}, \mathrm{b}, 1}$, Alex Lewis ${ }^{\mathrm{a}, 2}$ \\ a Department of Mathematical Physics, National University of Ireland, Maynooth, Republic of Ireland \\ ${ }^{\mathrm{b}}$ Dublin Institute for Advanced Studies, 10 Burlington Rd., Dublin 4, Republic of Ireland
}

Received 17 May 1999

Editor: P.V. Landshoff

\begin{abstract}
A family of connections on the space of couplings for a renormalizable field theory is defined. The connections are obtained from a Levi-Civita connection, for a metric which is a generalisation of the Zamolodchikov metric in two dimensions, by adding a family of tensors which are solutions of the renormalization group equation for the operator product expansion co-efficients. The connections are torsion free, but not metric compatible in general. The renormalization group flows of $N=2$ supersymmetric Yang-Mills theory in four dimensions and the $O(N)$-model in three dimensions, in the large $N$ limit, are analysed in terms of parallel transport under these connections. (C) 1999 Published by Elsevier Science B.V. All rights reserved.
\end{abstract}

In this letter we investigate geometrical properties of the renormalization group flow in some exactly solved theories. The renormalization group flow can be seen as a vector flow in the space of theories, with the couplings of the theory $g^{a}$ being coordinates on this space. In this approach, it has been shown in [1] following a suggestion in [2] (see also $[3,4]$ that the renormalization group equations for multi-point correlation functions, written in a coordinate covariant form, depend on a symmetric connection $\Gamma_{b c}^{a}$ through a tensor $\tau_{b c}^{a}$,

$\tau_{b c}^{a}=\nabla_{b} \nabla_{c} \beta^{a}-R_{c b d}^{a} \beta^{d}$,

\footnotetext{
${ }^{1}$ E-mail: bdolan@thphys.may.ie

${ }^{2}$ Supported by Enterprise Ireland grant no. SC/98/739: E-mail:alex@thphys.may.ie
}

defined by the RG equation for a regularized 3-point function $G_{a b c}(p, q, r)=\left\langle\Phi_{a}(p) \Phi_{b}(q) \Phi_{c}(r)\right\rangle$

$$
\begin{aligned}
\left(\Lambda \frac{\partial}{\partial \Lambda}+\mathscr{L}_{\beta}\right) G_{a b c}(p, q, r) \\
=\tau_{a b}^{d} G_{d c}(p+q, r)+\tau_{b c}^{d} G_{d a}(q+r, p) \\
\quad+\tau_{a c}^{d} G_{d b}(r+p, q)+\ldots,
\end{aligned}
$$

where $G_{a b}(p, q)=\left\langle\Phi_{a}(p) \Phi_{b}(q)\right\rangle$ and the dots denote contact terms that are only important for large momenta. However, there is no general rule for finding a connection. Moreover, since the RG equations only depend on the connection through the tensor $\tau_{b c}^{a}$, there is in fact a family of connections which give the same equations. The approach we take is therefore to determine the full family of possible connections for some exactly solvable models, and investigate the geometrical properties of the 
RG flow for the most general connection. For two connections $\Gamma$ and $\tilde{\Gamma}$ with covariant derivatives $\nabla$ and $\tilde{\nabla}$ and curvatures $R$ and $\tilde{R}$ respectively to both be compatible with Eq. (1) we must have

$$
\nabla_{b} \nabla_{c} \beta^{a}-R_{c b d}^{a} \beta^{d}=\tilde{\nabla}_{b} \tilde{\nabla}_{c} \beta^{a}-\tilde{R}_{c b d}^{a} \beta^{d} .
$$

This equation is satisfied if the Lie derivative $\mathscr{L}_{\beta}$ of the difference between the connections vanishes. This enables us to determine the full family of possible connections if one connection $\stackrel{o}{\Gamma}_{b c}^{a}$ is already known: we can write any connection which is compatible with Eq. (1) as $\Gamma_{b c}^{a}=\stackrel{o}{\Gamma}_{b c}^{a}+\mathscr{G}_{b c}^{a}$, where

$$
\begin{aligned}
\mathscr{L}_{\beta} & \mathscr{G}_{b c}^{a} \\
& =\mathscr{G}_{d c}^{a} \partial_{b} \beta^{d}+\mathscr{G}_{b d}^{a} \partial_{c} \beta^{d}-\mathscr{G}_{b c}^{d} \partial_{d} \beta^{a}+\beta^{d} \partial_{d} \mathscr{G}_{b c}^{a} \\
& =0 .
\end{aligned}
$$

We still have to find a connection $\stackrel{\mathrm{o}}{\Gamma}_{b c}^{a}$ to construct the other possible connections. One solution is to use the Levi-Civita connection of a metric on the space of couplings. An example of such a metric is the Zamolodchikov metric in $D=2$, which was used in the proof of the $c$-theorem [5]. More recently, building on ideas laid out in [3], the geometrical properties of metrics in $D>2$ have also been investigated for some models, including: free field theory [6], the $\mathrm{O}(\mathrm{N})$ model [7] and Seiberg-Witten theory for $S U(2)$ [8]. In all these models, it was found that some (but not all) of the RG flow lines are geodesics of the metric. In particular, the lines of crossover between fixed points are geodesics, and this may be related to irreversibility of the renormalization group flow. Since we now have a family of connections which are equivalent, at least as far as the renormalization group equations are concerned, it is natural to ask whether the geodesic flow of the lines of crossover generalises to auto-parallel flow for other connections (a line which is auto-parallel for the Levi-Civita connection is a geodesic) ${ }^{3}$.

The auto-parallel equation for a vector field is $\nabla_{\boldsymbol{\beta}} \boldsymbol{\beta}=\eta \boldsymbol{\beta}$, where $\eta$ is a function which depends on

\footnotetext{
${ }^{3}$ We distinguish between auto-parallels, which are curves whose tangent vectors remain tangent vectors under parallel transport along the curve, and geodesics, which are curves of shortest length. In general, these coincide for the Levi-Civita connection only.
}

the parameterization along the curve. With a connection $\Gamma=\stackrel{\circ}{\Gamma}+\mathscr{G}$, this becomes

$\beta^{b} \frac{\partial \beta^{a}}{\partial x^{b}}+\stackrel{\mathrm{o}}{\Gamma}_{b c}^{a} \beta^{b} \beta^{c}+\mathscr{G}_{b c}^{a} \beta^{b} \beta^{c}=\eta \beta^{a}$.

Our main aim is to see which of the possible connections, if any, will satisfy this equation for a given renormalization group trajectory. In particular, some of the trajectories in the models we will examine in this letter are geodesics of the metric, so Eq. (5) is satisfied for $\mathscr{G}=0$. In that case, Eq. (5) simplifies to the condition that

$\mathscr{G}_{b c}^{a} \beta^{b} \beta^{c}=\eta^{\prime} \beta^{a}$,

where $\eta^{\prime}$ is another function.

A natural candidate for a metric on the space of couplings is the two-point correlation functions of the model [3]. If the action $S$ is linear in the couplings,

$S=S_{0}+\int d^{D} x g^{a} \Phi_{a}(x)$

then a metric can be defined by

$G_{a b}=\int d^{D} x\langle\tilde{\Phi}(x) \tilde{\Phi}(0)\rangle$,

where $\tilde{\Phi}(x)=\Phi(x)-\langle\Phi(x)\rangle$. Although the individual components of this metric may diverge, the geometry can still be non-singular.

As our first example, we consider the $O(N)$ model for large $N$ in 3 dimensions. This is a model of a scalar field $\boldsymbol{\varphi}$ in the vector representation of $O(N)$ with the action

$S=\int d^{3} x\left\{\frac{1}{2}(\nabla \boldsymbol{\varphi})^{2}+\boldsymbol{j} \cdot \boldsymbol{\varphi}+\frac{r}{2} \boldsymbol{\varphi}^{2}+\frac{u}{4 !}\left(\boldsymbol{\varphi}^{2}\right)^{2}\right\}$

following [7] we analyse the geometry in terms of three bare parameters, $\phi, X, \lambda$, defined by

$\phi=4 \sqrt{\frac{\pi}{N \Lambda}}\langle\varphi\rangle, \quad X=\frac{1}{2 \Lambda} \int d^{3} x\left\langle\varphi^{2}\right\rangle$,

$\lambda=\frac{N u}{48 \pi \Lambda}$.

Although these are bare parameters, they are finite as we have a finite cut-off $\Lambda$, so we can use them as 
our coordinates on the space of couplings (since we are in any case only interested in properties of the RG flow which are independent of the coordinate system). The beta functions, which represent a vector flow on this space are [7]

$\beta^{\phi}=-\frac{1}{2} \phi, \quad \beta^{X}=-X, \quad \beta^{\lambda}=-\lambda$.

In [7] the metric (8) was computed, and it was found that only one of the renormalization group trajectories described by these beta functions is actually a geodesic of the metric - the line $X=\phi=0$, which is the line of crossover from the Wilson-Fisher fixed point at $\lambda=\infty$ to the Gaussian fixed point at $\lambda=0$. We now want to see if any of the renormalization group trajectories are auto-parallel for a connection $\Gamma=\stackrel{\mathrm{o}}{\Gamma}+\mathscr{G}$, where $\stackrel{\mathrm{o}}{\Gamma}$ is the Levi-Civita connection from [7] and $\mathscr{G}$ is a solution of Eq. (4). Using bare rather than renormalized parameters as coordinates makes it easy to solve Eq. (4) - the general solution contains a number of arbitrary functions which depend only on the ratios $X / \lambda$ and $\phi^{2} / \lambda$, but these ratios are constant along any of the $\mathrm{RG}$ trajectories described by Eq. (11), so we can treat them as just being arbitrary constants, which we write as $f_{j k}^{i}$. The solution can then be written, provided $X, \phi$ and $\lambda$ are non-zero, as

$\mathscr{G}_{j k}^{i}=\frac{\beta^{i}}{\beta^{j} \beta^{k}} f_{j k}^{i}$

(no summation on $i, j, k$ ). With this solution, it is clear that for Eq. (5) to be satisfied for a trajectory, the following differences must be constant along the curve:

$$
\begin{aligned}
& \frac{2}{\phi} \stackrel{\mathrm{o}}{\Gamma}_{i j}^{\phi} \beta^{i} \beta^{j}-\frac{1}{\lambda} \stackrel{\mathrm{o}}{\Gamma}_{i j}^{\lambda} \beta^{i} \beta^{j}=\text { constant, } \\
& \frac{2}{\phi} \stackrel{\mathrm{o}}{\Gamma}_{i j}^{\phi} \beta^{i} \beta^{j}-\frac{1}{X} \stackrel{\mathrm{o}}{\Gamma}_{i j}^{X} \beta^{i} \beta^{j}=\text { constant. }
\end{aligned}
$$

It can be seen from the expressions for the components of the Levi-Civita connection given in Appendix 2 of [6] that this is not true for any of the RG flow lines except the line of crossover. Thus none of the flow lines with $\phi, X$, and $\lambda$ all non-zero can be auto-parallel for any connection. When one of $X, \phi$ or $\lambda$ is 0 , the solution (12) has to be changed by absorbing factors of $X / \lambda$ or $\phi^{2} / \lambda$ into $f_{j k}^{i}$ to make it finite, for example if $\lambda \neq 0$ we can write the solution as

$\mathscr{G}_{j k}^{i}=\lambda^{n} f_{j k}^{i}, \quad n=n_{i}-n_{j}-n_{k}$

with $n_{\phi}=\frac{1}{2}$ and $n_{X}=n_{\lambda}=1$. However, if $X=0$, $\phi=0$ or $\lambda=0$, Eq. (5) can only be satisfied if $\stackrel{\circ}{\Gamma}_{i j}{ }^{X} \beta^{i} \beta^{j}=0, \quad \stackrel{\circ}{\Gamma}_{i j} \beta^{i} \beta^{j}=0$ or $\stackrel{\circ}{\Gamma}_{i j}^{\lambda} \beta^{i} \beta^{j}=0$ respectively, and the only line which satisfies these conditions is $X=\phi=0$. Thus none of the other RG flow lines can ever be auto-parallel. Finally, we want to see if the geodesic $X=\phi=0$ is auto-parallel for other connections (apart from the Levi-Civita connection). This means we have to see if Eq. (6) is satisfied by the solution (14). For example, if only $f_{\phi \phi}^{\phi}, f_{\lambda \lambda}^{\lambda}$ and $f_{X X}^{X}$ are non-zero, Eq. (6) becomes

$$
\begin{aligned}
& -\eta^{\prime} \phi=\lambda^{-1 / 2} f_{\phi \phi}^{\phi} \phi^{2} / 2, \quad-\eta^{\prime} \lambda=\lambda^{-1} f_{\lambda \lambda}^{\lambda} \lambda^{2}, \\
& -\eta^{\prime} X=\lambda^{-1} f_{X X}^{X} X^{2},
\end{aligned}
$$

which clearly is satisfied for $X=\phi=0$. However this is not true for the most general connection. For example, if $\mathscr{G}_{\lambda \lambda}^{X}=\lambda^{-1} f_{\lambda \lambda}^{X} \neq 0$ or $\mathscr{G}_{\lambda \lambda}^{\phi}=\lambda^{-3 / 2} f_{\lambda \lambda}^{\phi} \neq 0$ then the line of crossover is not an auto-parallel (since the corresponding Levi-Civita connection components vanish in the large $N$ limit [7]). The line of crossover is therefore not auto-parallel for the entire family of connections which can be used in the RG equations, but only for the class with $\mathscr{G}_{\lambda \lambda}^{X}=$ $\mathscr{G}_{\lambda \lambda}^{\phi}=0$.

Thus we find that introducing the family of nonmetric compatible connections changes the conclusions, compared to the the Levi-Civita case, as to which renormalization group flow lines are autoparallel. There is a large class of connections for which the line of crossover between the Gaussian and the Wilson-Fisher fixed points remains autoparallel, but this is not true of the most general connection.

Our second example of an RG flow shows that, in general, the family of connections do change the auto-parallel nature of the geodesic flows (in the special cases where RG flow is geodesic). This is 4-dimensional $N=2$ Yang-Mills theory [9]. The geometrical properties of this model were investigated in [8] where two possible metrics were considered, and it was found most of the renormalization group flow lines are not geodesics, but some special lines are geodesics of both metrics. We now want to see 
which, if any, of the RG flow lines are auto-parallel for other connections. The complex coupling $\tau=\frac{\theta_{\text {eff }}}{2 \pi}$ $+\frac{4 \pi i}{g_{\text {eff }}^{2}}$ is given as a function of $\tilde{u}=u / \Lambda^{2}$, where $u=\operatorname{Tr}\left\langle\varphi^{2}\right\rangle$ parameterizes the symmetry breaking, by

$\tau=\frac{i K^{\prime}}{K}+2 n$

where $K\left(k^{2}\right)$ is a standard elliptic integral [10] with $k^{2}=\frac{2}{\tilde{u}+1}$ and $K^{\prime}=K\left(1-k^{2}\right)$ and $n$ is an integer. Various beta functions for this model have been investigated in [11-13]. In [13] Wilsonian and Novikov, Shifman, Vainshtein, Zakharov [14], beta functions were considered. Here we concentrate on the Callan-Symanzik beta functions of $[11,12]$. The Callan-Symanzik beta functions, $\boldsymbol{\beta}=\beta \frac{\partial}{\partial \tau}+\bar{\beta} \frac{\partial}{\partial \bar{\tau}}$ are defined by

$\beta(\tau)=\left.\lambda \frac{\partial \tau}{\partial \Lambda}\right|_{u}=-2 \tilde{u} \frac{d \tau}{d \tilde{u}}$.

This beta function represents a vector flow on a manifold, parameterized by $\tau$ (or $u$ ), which has the topology of a sphere with three punctures. This manifold has three singular points: $u=\infty$ (the weak coupling limit) and $u= \pm \Lambda^{2}$ (where there are extra massless degrees of freedom). The Seiberg-Witten metric on this manifold in the $u$-coordinates is

$$
\begin{aligned}
d s^{2} & =\pi^{2} \operatorname{Im}(\tau)\left|\frac{\vartheta_{3}^{4} \vartheta_{4}^{4}}{\vartheta_{2}^{2}}\right|^{2} d \tau d \bar{\tau} \\
& =\frac{1}{\pi^{2}} \frac{\left(K^{\prime} \bar{K}+\bar{K}^{\prime} K\right)}{\sqrt{1+u} \sqrt{1+\bar{u}}} d u d \bar{u},
\end{aligned}
$$

where $\vartheta_{i}, i=2,3,4$ are Jacobi $\vartheta$-functions, [10].

Another metric which can be introduced for this geometry is the Poincaré metric

$d s^{2}=\frac{1}{(\operatorname{Im}(\tau))^{2}} d \tau d \bar{\tau}$.

For both these metrics, the lines of real $u$ and imaginary $u$ are geodesics, but the other RG flow lines are not [8].

In fact we do not need to know the explicit form of $\beta(\tau)$ to see if the geodesics will be auto-parallel for any connection: if we use $u$ and $\bar{u}$ as coordinates, so that $\boldsymbol{\beta}=\beta(u) \frac{\partial}{\partial u}+\bar{\beta}(u) \frac{\partial}{\partial \bar{u}}$, we only need to know that $\frac{\partial \beta}{\partial \bar{u}}=\frac{\partial \bar{\beta}}{\partial u}=0$. The solution of Eq. (4) is then

$$
\begin{array}{rlrl}
\mathscr{G}_{u u}^{u} & =\frac{1}{\beta} g_{u u}^{u}(u / \bar{u}), & \mathscr{G}_{\bar{u} \bar{u}}^{\bar{u}}=\frac{1}{\bar{\beta}} g_{\bar{u} \bar{u}}^{\bar{u}}(u / \bar{u}), \\
\mathscr{G}_{\bar{u} u}^{u}=\frac{1}{\bar{\beta}} g_{\bar{u} u}^{u}(u / \bar{u}), & \mathscr{G}_{u \bar{u}}^{\bar{u}}=\frac{1}{\beta} g_{u \bar{u}}^{\bar{u}}(u / \bar{u}), \\
\mathscr{G}_{\bar{u} \bar{u}}^{u}=\frac{\beta}{\bar{\beta}^{2}} g_{\bar{u} \bar{u}}^{u}(u / \bar{u}), & \mathscr{G}_{u u}^{\bar{u}}=\frac{\bar{\beta}}{\beta^{2}} g_{u u}^{\bar{u}}(u / \bar{u}) .
\end{array}
$$

The solution contains arbitrary functions of $u / \bar{u}$, but these functions are constant along the radial lines in the $u$-plane, and we can see from Eq. (17) that these are just the RG flow lines (in the $u$-coordinates, $\left.\beta^{u}=-2 u, \beta^{\bar{u}}=-2 \bar{u}\right)$. If we assume that $\mathscr{G}=$ $\mathscr{G}_{j k}^{i} d x^{j} \otimes d x^{k} \otimes \frac{\partial}{\partial x^{i}}$ is a real tensor and substitute this solution into Eq. (5) or (6), we can see that those equations depend only on one function $g(u / \bar{u})$ :

$g(u / \bar{u})=g_{u u}^{u}+g_{\bar{u} u}^{u}+g_{u \bar{u}}^{u}+g_{\bar{u} \bar{u}}^{u}$,

$\bar{g}(u / \bar{u})=g_{\bar{u} \bar{u}}^{\bar{u}}+g_{u \bar{u}}^{\bar{u}}+g_{\bar{u} u}^{\bar{u}}+g_{u u}^{\bar{u}}$.

Eq. (5) then reduces to the condition that

$\stackrel{\circ}{\Gamma}_{u u}^{u} u+g(u / \bar{u})=\stackrel{\circ}{\Gamma_{\bar{u}}} \bar{u} \bar{u}+\bar{g}(u / \bar{u})$

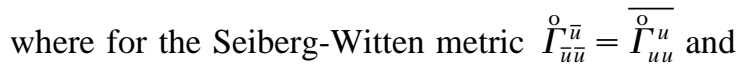

$\stackrel{\circ}{\Gamma}_{u u}^{u}=\frac{\bar{K}^{\prime} \partial_{u} K+\bar{K} \partial_{u} K^{\prime}}{K^{\prime} \bar{K}+\bar{K}^{\prime} K}-\frac{1}{2(u+1)}$.

Since $g$ and $\bar{g}$ are constant along an RG trajectory, a trajectory can only be auto-parallel for some choice of $g$ and $\bar{g}$ if the imaginary part of $\stackrel{0}{\Gamma}_{u u}^{u} u$ is constant along that trajectory. It can be shown numerically that this is only the case for the geodesics, the lines of real and imaginary $u$, where $\stackrel{\circ}{\Gamma}_{u u}^{u} u$ is real. All the other RG flow lines are therefore not auto-parallel for any connection, while the real $u$ line is autoparallel provided $g(1)=\bar{g}(1)$ and the imaginary $u$ line is auto-parallel provided $g(-1)=\bar{g}(-1)$. Thus the geodesic flow lines are not auto-parallel for the most general connection (as was the case in the $O(N)$ model), but they are auto-parallel, for example, if $\bar{g}(u / \bar{u})=g(\bar{u} / u)$. 
Since we did not use the explicit expressions for $\tau$ and $\beta$ above, this result can be applied to any theory in which the beta function is an analytic function of one complex variable only. In the case of $N=2$ $S U(2)$ Yang-Mills theory with massless quarks, as in the pure Yang-Mills theory, the complex coupling $\tau$ depends only on $u / \Lambda^{2}$. However, the singularities of the theories with massless quarks are not all on the real or imaginary $u$ axes - for example, when the number of flavours $N_{f}=1$, there are singularities at $u=-u_{0}$ and $u=u_{0} e^{ \pm i \pi / 3}$. Of course, we cannot tell if these lines are actually auto-parallel unless we know whether they are geodesics of the Seiberg-Witten metric, but we can say that if they are geodesics of the Seiberg-Witten metric in these models, they be auto-parallel for connections with $g\left(e^{2 \pi i / 3}\right)=$ $\bar{g}\left(e^{2 \pi i / 3}\right)$, but not for all connections for which $\bar{g}(u / \bar{u})=g(\bar{u} / u)$, as in the case of the pure YangMills theory.

In conclusion it has been shown that the renormalization group equation for the operator expansion co-efficients gives rise to a family of non-metric compatible connections which are related to the Levi-Civita connection by $\Gamma_{b c}^{a}=\stackrel{\circ}{\Gamma}_{b c}^{a}+\mathscr{G}_{b c}^{a}$, where $\mathscr{L}_{\beta} \mathscr{G}_{b c}^{a}=0$. In general RG flows which are geodesic for the Levi-Civita connection are not auto-parallel for all members of the family, though in the models examined they are for a large sub-class of connections (it can be shown that the same is also true for the free field models considered in [6]). In none of the examples examined here is it the case that a $R G$ flow line which is not geodesic under the Levi-Civita connection is auto-parallel for some member of the family.

\section{References}

[1] B.P. Dolan, Int. J. Mod. Phys. A 10 (1995) 2439, hepth/9403070.

[2] I. Jack, H. Osborn, Nucl. Phys. B 343 (1990) 647.

[3] D. O'Connor, C.R. Stephens, Geometry, the renormalization group and gravity, in Directions in General Relativity - Proc. 1993 Int. Symp. Maryland, eds. B.L. Hu, M.P. Ryan Jr., C.V. Vishevshawara, Cambridge University Press (1993), Vol. 1.

[4] H. Sonoda, Nucl. Phys. B 383 (1992) 173; Nucl. Phys. B 408 (1993) 180, B 394 (1993) 302; hep-th/9306119; K. Ranganathan, hep-th/9210090.

[5] A.B. Zamolodchikov, Pis'ma Zh. Eksp. Teor. Fiz. 43 (1986) 565.

[6] B.P. Dolan, Int. J. Mod. Phys. A 12 (1997) 2413, hepth/9511175.

[7] B.P. Dolan, Nucl. Phys. B 528 (1998) 553, hep-th/9702156.

[8] B.P. Dolan, Phys. Lett. B 418 (1998) 107, hep-th/9710161.

[9] N. Seiberg, E. Witten, Nucl. Phys. B 426 (1994) 19, hepth/9407087.

[10] E.T. Whittaker, G.N. Watson, A course of Modern Analysis, Cambridge University Press (1927).

[11] G. Bonelli, M. Matone, Phys. Rev. Lett. 76 (1996) 4107, hep-th/9602174.

[12] A. Ritz, Phys. Lett. B 434 (1998) 54, hep-th/9710112.

[13] G. Carlino, K. Konishi, N. Maggiore, N. Magnoli, hepth/9902162.

[14] V.A. Novikov, M.A. Shifman, A.I. Shifman, V.I. Zakharov, Nucl. Phys. B 229 (1983) 381; ibid. B 260 (1985) 157; Phys. Lett. B 166 (1986) 334. 